\title{
THE CONTINUITY OF FUNCTIONS ON CARTESIAN PRODUCTS
}

\author{
BY \\ N. NOBLE( $\left.{ }^{1}\right)$
}

Introduction. $\mathrm{A}$ function $f$ is sequentially continuous if the restriction of $f$ to each convergent sequence (including its limit) is continuous, and $f$ is $k$-continuous if its restriction to each compact subspace is continuous. If each sequentially continuous (resp. real valued sequentially continuous) function with domain $X$ is continuous, $X$ is a sequential space (resp. $s_{R}$-space); $k$-spaces and $k_{R}$-spaces are defined analogously.

In this paper we are concerned with determining conditions under which sequentially continuous or $k$-continuous functions on a product space $X=\prod_{\alpha \in A} X_{\alpha}$ will be continuous. Concerning sequentially continuous functions, our result includes conditions necessary and sufficient that a product of first countable spaces be a sequential space or an $s_{R}$-space. Concerning $k$-continuous functions, we show that if each $X_{\alpha}$ is either first countable or locally compact, then each $k$ continuous function on $X$ with regular range is continuous, and that products of locally pseudocompact $k_{R}$-spaces are $k_{R}$-spaces. We also consider sequentially continuous and $k$-continuous group homomorphisms, and show, for instance, that the property "each $k$-continuous homomorphism with $T_{0}$ range is continuous" is preserved under arbitrary products.

All of these results are given in $\$ 5$. $\$ 1$ presents three fairly general conditions which can be combined to force the continuity of functions on product spaces, and these conditions are studied in $\S \S 2,3$ and 4 . As incidental results we answer negatively a pair of questions: "Is the Hewitt-Nachbin realcompactification of a Fréchet space a $k$-space?" and "If $X$ and $Y$ are normal $k$-spaces, is the $k$-extension of $X \times Y$ completely regular?" raised by W. W. Comfort and E. A. Michael respectively ( $\$ 2)$; answer a question posed by Keisler and Tarski in [10] by showing that a certain condition on cardinals is equivalent to measurability ( $\$ 3)$; and prove an analogue of Tychonoff's Theorem by showing that $k$-compactness (introduced in [4]) is preserved under arbitrary products (\$4).

1. Conditions forcing continuity. Recall that a subspace of $X=\prod_{\alpha \in A} X_{\alpha}$ is called a $\Sigma$-subspace if it has the form $\{x \in X: \delta(x, y)$ is countable $\}$ for some fixed $y$ in $X$,

Received by the editors January 8, 1969.

( ${ }^{1}$ This paper is an expanded version of the fourth chapter of the author's doctoral dissertation, which was written at the University of Rochester under the direction of $\mathrm{W}$. W. Comfort. 
where $\delta(x, y)=\left\{\alpha: x_{\alpha} \neq y_{\alpha}\right\}$. (Such subspaces have been studied in [3].) Call a $\Sigma$-subspace a $\Sigma^{0}$-subspace if in fact each $\delta(x, y)$ is finite, and call a function defined on a product space $\Sigma$-continuous (resp. $\Sigma^{0}$-continuous) if its restriction to each $\Sigma$-subspace (resp. $\Sigma^{0}$-subspace) is continuous. Also, call a function 2-continuous if it is continuous when restricted to each subspace of the form $\prod_{\alpha \in A} Y_{\alpha}$ where for each $\alpha, 1 \leqq \operatorname{card}\left(Y_{\alpha}\right) \leqq 2$.

1.1 TheOREM. Let $Z$ be regular. If $f: \prod_{\alpha \in A} X_{\alpha} \rightarrow Z$ is $\Sigma^{0}$-continuous and 2continuous, then $f$ is continuous.

Proof. Let $U$ be an open subset of $Z$, let $x$ be a point in $f^{-1}(U)$ and let $V$ be an open neighborhood of $x$ such that cl $V \subseteq U$. Let $Y$ be the $\Sigma^{0}$-subspace which contains $x$; since $f^{-1}(V) \cap Y$ is open in $Y$, there exists a finite subset $F$ of $A$ and neighborhoods $V_{\alpha}$ of $x_{\alpha}$ such that for $W=\prod_{\alpha \in F} V_{\alpha} \times \prod_{\alpha \in A \mid F} X_{\alpha}, W \cap Y \subseteq f^{-1}(V)$.

Now suppose there exists a point $y$ in $W$ such that $f(y) \notin U$, set $Y^{\prime}=\prod_{\alpha \in A}\left\{x_{\alpha}, y_{\alpha}\right\}$ and note that, since $f$ is 2-continuous, there exists a finite $F^{\prime}$ such that for $W^{\prime}=\prod_{\alpha \in F^{\prime}}\left\{y_{\alpha}\right\} \times \prod_{\alpha \in A \mid F^{\prime}}\left\{x_{\alpha}, y_{\alpha}\right\}, W^{\prime} \subseteq f^{-1}(X \mid c l V)$. Since $W^{\prime} \cap W$ is clearly not empty, this is impossible, so $f(W) \subseteq U$ and hence $f$ is continuous.

Call $f \Sigma$-semicontinuous (resp. $\Sigma^{0}$-semicontinuous) if whenever $U$ is open and $x \in f^{-1}(U)$, there exists a finite $F \subseteq A$ such that $\Sigma_{F}(x) \subseteq f^{-1}(U)\left(\operatorname{resp} . \Sigma_{F}^{0}(x) \subseteq f^{-1}(U)\right)$ where $\Sigma_{F}(X)=\{y: \delta(x, y)$ is countable and disjoint from $F\}$, and $\Sigma_{F}^{0}(X)=$ $\{y: \delta(x, y)$ is finite and disjoint from $F\}$. Recall that a map is closed if it carries closed sets to closed sets, and let $\pi_{B}$ denote the projection: $\prod_{\alpha \in A} X_{\alpha} \rightarrow \prod_{\alpha \in B} X_{\alpha}$.

1.2 TheOREM. Let $Z$ be regular. If $f: \prod_{\alpha \in A} X_{\alpha} \rightarrow Z$ is 2-continuous and $\Sigma^{0}$ semicontinuous, and if $\pi_{F} \circ f^{-1}$ is closed for each finite $F \subseteq A$, then $f$ is continuous.

Proof. Given $x \in f^{-1}(U)$ with $U$ open, choose $V$ open with $x \in V$ and $\operatorname{cl} V \subseteq U$ and let $F \subseteq A$ be finite such that $\Sigma_{F}^{0}(x) \subseteq f^{-1}(V)$. Let $X^{\prime}=\pi_{F}(X), X^{\prime \prime}=\pi_{A \mid F}(X)$ and $x^{\prime}=\pi_{F}(x)$ and note that, as in the proof of $1.1, x^{\prime} \times X^{\prime \prime} \subseteq f^{-1}(U)$. Thus $x^{\prime}$ is not in the closed set $S=\pi_{F} \circ f^{-1}(Z \backslash U)$, so $x \in\left(X^{\prime} \mid S\right) \times X^{\prime \prime} \subseteq f^{-1}(U)$. Therefore $f$ is continuous.

Note that the proof actually shows: If $f: \prod_{\alpha} X_{\alpha} \rightarrow Z$ is $\Sigma^{0}$-semicontinuous and for each $\Sigma^{0}$-subspace $Y$ of $\prod_{\alpha} X_{\alpha},\left.\left.\pi_{F}\right|_{Y} \circ f\right|_{Y} ^{-1}$ is closed for each finite subset $F$ of $A$, then $f$ is $\Sigma^{0}$-continuous. By the obvious adaptation of our proof, the corresponding result for $\Sigma$-semicontinuous functions and $\Sigma$-subspaces holds.

For conditions under which $\pi_{F} \circ f^{-1}$ will be closed, see [6], [9], [16] or [17].

Where $G=\prod_{\alpha} G_{\alpha}$ with each $G_{\alpha}$ a topological group, we consider subproducts of $G$ to be embedded in $G$ in the natural way (i.e., $\prod_{\alpha \in B} G_{\alpha}=\prod_{\alpha \in B} G_{\alpha} \times \prod_{\alpha \in A \mid B}\left\{e_{\alpha}\right\}$ where $e_{\alpha}$ is the identity of $G_{\alpha}$ ). A subgroup $H$ of $G$ is invariant under projections if $\pi_{B}(H) \subseteq H$ for each $B \subseteq A$. Note that the $\Sigma^{0}$-subgroup (=the direct sum of the $G_{\alpha}$ ), the $\Sigma$-subgroup, and of course $G$, are invariant under projections.

1.3 THEOREM. Let $G=\prod_{\alpha} G_{\alpha}$ where each $G_{\alpha}$ is a topological group, let $H$ be a subgroup of $G$ which is invariant under projections, and let $\psi$ be a homomorphism 
on $H$ with $T_{0}$ range. If $\psi$ is separately continuous (continuous on each factor) and $\Sigma^{0}$-semicontinuous (resp. $\Sigma$-semicontinuous) then $\psi$ is $\Sigma^{0}$-continuous (resp. $\Sigma$-continuous).

Proof. Let us first note that, like any separately continuous homomorphism (since for $\psi(x) \in V$ and $\left.V^{n} \subseteq U, \psi\left(\prod_{i=1}^{n} \psi^{-1}(V) \cap G_{i}\right) \subseteq U\right), \psi$ is continuous on finite products. For $x \in f^{-1}(U)$ and $V$ such that $V^{2} \subseteq U$ let $F \subseteq A$ be finite such that $\Sigma_{F}^{0}(x) \subseteq \psi^{-1}(V)$ and let $V^{\prime}=\psi^{-1}(V) \cap \prod_{\alpha \in F} X_{\alpha}$. By the comment above, $V^{\prime}$ is open; since $\psi\left(V^{\prime} \cdot \Sigma_{F}^{0}(x)\right) \subseteq \psi\left(V^{\prime}\right) \cdot \psi\left(\Sigma_{F}^{0}(x)\right) \subseteq V^{2} \subseteq U$, this shows that $\psi$ is $\Sigma^{0}$ continuous. The obvious adaptation proves the remaining statement.

2. $\Sigma$-subspaces. A space $X$ is called a Fréchet space if whenever $x$ is in the closure of $S \subseteq X, S$ contains a sequence which converges to $x$. Each Fréchet space is sequential and each subspace of a Fréchet space is a Fréchet space-indeed, a space is hereditarily sequential if and only if it is a Fréchet space. Our first result generalizes the relevant portions of [12, Theorem II] and [5, Theorem 3].

2.1 THEOREM. Each $\Sigma$-subspace of a product of first countable spaces is a Fréchet space.

Proof. Let $Y$ be a $\Sigma$-subspace of $\prod_{\alpha \in A} X_{\alpha}$ where each $X_{\alpha}$ is first countable, let $S \subseteq Y$ and let $x$ be any point in the closure of $S$. For each $\alpha$ let $\left\{U_{\alpha}^{n}: n=1,2, \ldots\right\}$ be a nested base for the neighborhoods of $x_{\alpha}$. Choose $y^{\prime}$ in $S$ and, inductively, choose $y^{n} \in S \cap \prod_{\beta \in B(n)} U_{\beta}^{n} \times \prod_{\beta \in A \mid B(n)} X_{\beta}$ where $B(n)=\left\{\alpha_{j}^{i}: 1 \leqq i, j<n\right\}$ and the $\alpha_{j}^{i}$ are determined by index $\delta\left(x, y^{i}\right)$ as $\left\{\alpha_{j}^{i}: i=1,2, \ldots\right\}$. Such points $y^{n}$ can always be chosen since neighborhoods of $x$ must meet $S$.

We complete the proof by showing that $\left\{y^{n}\right\}$ converges to $x$. Let $U$ be any neighborhood of $x$ and, without loss of generality, suppose $U=\left(\prod_{\alpha \in F} U_{\alpha}^{m} \times \prod_{\alpha \in A \mid F} X_{\alpha}\right)$ $\cap Y$ for some integer $m$ and some finite subset $F$ of $A$. Since $F$ is finite and $\{(B n)\}$ are increasing, there exists an integer $n_{0}$ such that $F \cap U_{n} B(n) \subseteq B\left(n_{0}\right)$. Since for $\alpha \notin U_{n} B(n), y_{\alpha}^{n}=x_{\alpha}, y^{n}$ is in $U$ whenever $n>\max \left\{n_{0}, m\right\}$, so $\left\{y^{n}\right\}$ converges to $x$.

2.2 Corollary. Each sequentially continuous function on $\prod_{\alpha} X_{\alpha}$ is $\Sigma$-semicontinuous.

Proof. Retopologize each $X_{\alpha}$ to be discrete and apply 2.1.

Incidentally, Theorem 2.1 provides examples answering questions raised by W. W. Comfort and E. A. Michael in the context of [2] and [13] respectively. Comfort asked if the Hewitt-Nachbin realcompactification $\nu Y$ of a Fréchet space $Y$ is a $k$-space; the answer is no since if $X$ is a product of uncountably many copies of the reals, and $Y$ is any $\Sigma$-subspace of $X$, then $\nu Y=X$ [3, Theorem 2] but $X$ is not a $k$-space [11, p. 240]. Michael's question was: “Is the $k$-extension of $X \times Y$ completely regular when $X$ and $Y$ are $k$-spaces and are $<$ completely regular, normal, paracompact, $\boldsymbol{\aleph}_{0}$-spaces $>$ ? We answer the first two: Let $Y^{\prime}$ be a product of uncountably many copies of the unit interval, and let $Y$ be a $\Sigma$-subspace of $Y^{\prime}$. Since $Y$ is not locally compact, there exists [14, Theorem 3.1] a paracompact 
Hausdorff (hence normal) $k$-space $X$ such that $X \times Y$ is not a $k$-space. Also, $Y$ is normal by [3, Theorem 1]. Since $\nu Y=Y^{\prime}$ is compact, $Y$ is pseudocompact, so $X \times Y$ is a $k_{R}$-space [18, Theorem 4]. It follows that the $k$-extension of $X \times Y$ (the smallest topology on $X \times Y$ which makes $X \times Y$ a $k$-space and coincides with the product topology on compact sets) cannot be completely regular.

Note that (by Theorem 2.1) each $\Sigma$-subspace of $\prod_{\alpha \in A} X_{\alpha}$ will be a Fréchet space if each $X_{\alpha}$ is itself a $\Sigma$-subproduct of a product of first countable spaces. One cannot, however, allow the factors to be arbitrary Fréchet spaces: Let $X_{0}$ denote the quotient formed by identifying the limits of a countable collection of disjoint convergent sequences. Then $X_{0}$ is a Fréchet space, but (as in the proof of [20, Theorem 6.1]) $X_{0} \times Y$ is a Fréchet space, for $Y$ any $T_{1}$ space, if and only if $Y$ is discrete. It follows that if $X$ is an infinite product of $T_{1}$-spaces, one of whose factors contains a copy of $X_{0}$, then no $\Sigma$-subspace of $X$ is a Fréchet space.

Of course we do not really need $\Sigma$-subspaces to be Fréchet spaces. To apply Theorem 1.1 it suffices to know that certain functions on $\Sigma$-subspaces are continuous. Where $Y$ is any space, let $\mathscr{C}(Y)$ denote the collection of compact subsets of $Y$ which, as subsets, have countable neighborhood bases. (For $K \subseteq Y$ a neighborhood base for $K$ is a collection of open sets $U_{\alpha}$ such that for $V \supseteq K$ and $V$ open, $V \supseteq U_{\alpha} \supseteq K$ for some $\alpha$.) Let $\mathscr{C}^{*}(Y)=\{K \in \mathscr{C}(Y)$ : as a space, $K$ is first countable $\}$. A space $Y$ is said to be of pointwise countable type (we abbreviate this as "of type $\mathscr{C} ")$ if there is a subcollection $\mathscr{C}_{0}(Y)$ of $\mathscr{C}(Y)$ such that for each $y$ in $Y$, each $C$ in $\mathscr{C}_{0}$ with $y$ in $C$, and each neighborhood $U$ of $y$, there exists a $C^{\prime}$ in $\mathscr{C}_{0}$ with $y \in C^{\prime} \subseteq U \cap C$. We say that $Y$ is of type $\mathscr{C}^{*}$ if $\mathscr{C}_{0}(Y)$ can be chosen as a subcollection of $\mathscr{C}^{*}(Y)$.

Spaces of type $\mathscr{C}$ were introduced and studied by Arhangel'skiǐ in [1] where it is shown that locally compact $T_{2}$ spaces and spaces complete in the sense of Čech (absolute $G_{\delta}$ 's) and of course first countable spaces, are of this type. Arhangel'skii also proved the portion of the following proposition which applies to spaces of type $\mathscr{C}$.

2.3 Proposition. (i) Each space of type $\mathscr{C}$ is a $k$-space, and each space of type $\mathscr{C}^{*}$ is a sequential space.

(ii) Countable products of spaces of type $\mathscr{C}$ are of type $\mathscr{C}$ and countable products of spaces of type $\mathscr{C}^{*}$ are of type $\mathscr{C}^{*}$.

Proof. (i) Let $Y$ be of type $\mathscr{C}$, let $U \subseteq Y$ be $k$-open (i.e. $U \cap K$ is open in $K$ for each compact $K)$ and let $y \in U$. Choose $C^{\prime}$ in $\mathscr{C}_{0}(Y)$ with $y$ in $C^{\prime}$; since $U \cap C^{\prime}$ is open in $C^{\prime}$ there exists an open $U^{\prime}$ such that $U^{\prime} \cap C^{\prime}=U \cap C^{\prime}$ and hence, by the definition of spaces of type $\mathscr{C}$, there exists a $C$ in $\mathscr{C}$ with $y \in C \subseteq U^{\prime} \cap C^{\prime} \subseteq U$. Now suppose $U$ is not a neighborhood of $y$ and let $\left\{V^{n}\right\}$ be a base for the neighborhoods of $C$. Then for each $n, V^{n} \nsubseteq U$ so there exists a point $y^{n} \in V^{n} \backslash U$. Set $K=C \cup\left\{y^{n}: n=1,2, \ldots\right\}$. Clearly $K$ is compact, but since $U \cap K=C$ is not open in $K$, this yields a contradiction. 
Now let $Y$ be of type $\mathscr{C}^{*}$, and proceed as above when $U$ is sequentially open. Construct $K$ as before and note that $K$ is sequential so that $U \cap K$ should be (but is not) open.

(ii) This follows by the obvious construction.

2.4 TheOREM. Let $X=\prod_{\alpha \in A} X_{\alpha}$. If each $X_{\alpha}$ is of type $\mathscr{C}$, then each $k$-continuous function on $X$ is $\Sigma$-continuous. If each $X_{\alpha}$ is of type $\mathscr{C}^{*}$, then each $\Sigma$-subspace of $X$ is a sequential space (so each sequentially continuous function on $X$ is $\Sigma$-continuous).

Proof. Let $f$ be a $k$-continuous function on $X$, let $Y$ be a $\Sigma$-subspace of $X$, let $U$ be an open subset of the range of $f$, and let $y \in Y \cap f^{-1}(U)$. For each $\alpha$ choose $C_{\alpha} \in \mathscr{C}_{0}\left(X_{\alpha}\right)$ with $y_{\alpha} \in C_{\alpha}$, and note that, since $f^{-1}(U) \cap \prod_{\alpha} C_{\alpha}$ must be relatively open, we may suppose that $\prod_{\alpha} C_{\alpha}$ is contained in $f^{-1}(U)$. Now suppose $Y \cap f^{-1}(U)$ is not a neighborhood of $y$ in $Y$. Then, as in the proof of 2.1, we construct a sequence $\left\{y^{n}\right\} \leqq Y \backslash f^{-1}(U)$ which "converges" to $\prod_{\alpha} C_{\alpha}$. Let $K_{\alpha}=C_{\alpha} \cup\left\{y_{\alpha}^{n}\right\}$; then by construction each $K_{\alpha}$ must be compact, so $\left\{y^{n}\right\} \subseteq \prod_{\alpha} K_{\alpha}$ must have an accumulation point. Again by the construction, such an accumulation point must lie in $\prod_{\alpha} C_{\alpha}$, but this is impossible since $f^{-1}(U) \cap \prod_{\alpha} K_{\alpha}$ is open.

Now suppose each $X_{\alpha}$ is of type $\mathscr{C}^{*}$, let $Y$ be a $\Sigma$-subspace of $X$, let $U \subseteq Y$ be sequentially open and let $y$ in $U$. Choosing $C$ in $\mathscr{C}^{*}$ with $y_{\alpha}$ in $C_{\alpha}$ we may, by Theorem 2.1, suppose that $C=\prod_{\alpha} C_{\alpha} \cap Y$ is contained in $U$. If $U$ is not a neighborhood of $y$, then as before we construct $\left\{y^{n}\right\} \subseteq Y \backslash U$ which "converges" to $\prod_{\alpha} C_{\alpha}$, hence to $C$. Let $K=\operatorname{cl}\left(C_{\alpha} \cup\left\{y_{\alpha}^{n}\right\}\right)$ and let $B=\left\{\alpha: K_{\alpha} \neq C_{\alpha}\right\} ; B$ is countable so $\prod_{\alpha \in B} K_{\alpha}$ is sequential by 2.3 . Now $\left(\prod_{\alpha \in A \mid B} C_{\alpha}\right) \cap Y$ is sequential (by Theorem 2.1) and contains no infinite closed discrete subspace (since $\prod_{\alpha \in A \mid B} C_{\alpha}$ is compact) so by [18, Theorem 2], $\prod_{\alpha} K_{\alpha} \cap Y=\left(\prod_{\alpha \in B} K_{\alpha}\right) \times\left(\prod_{\alpha \in A \mid B} C_{\alpha} \cap Y\right)$ is sequential. But, as before, $U \cap\left(\prod_{\alpha} K_{\alpha} \cap Y\right)$ is not sequentially open and we have the desired contradiction.

Let us mention, although we do not wish to pursue it, that Theorems 2.3 and 2.4 remain true if, in the definition of spaces of type $\mathscr{C}^{*}$, one replaces the requirement that members of $\mathscr{C}$ be compact with the condition: Each countable subset has compact closure, or any other condition which insures countable compactness and is preserved under products.

3. Sequential cardinals. Theorems $2.1,2.3$ (and in fact 1.3) give conditions under which sequentially continuous functions will be $\Sigma$-continuous. In this section we investigate conditions under which a sequentially continuous function will be 2-continuous. As one would expect, such conditions involve only the cardinality of the index set $A$.

Let $A$ be a set; $2^{A}$ denotes the set of subsets of $A$. For $\left\{S_{n}\right\} \subseteq 2^{A}$, we use $\lim S_{n}=S$ to mean that $\left\{S_{n}\right\}$ converge to $S$ in the set-theoretic sense, i.e.,

$$
S=\bigcap_{n=1}^{\infty} \bigcup_{m=1}^{\infty} S_{n+m}=\bigcup_{n=1}^{\infty} \bigcap_{m=1}^{\infty} S_{n+m}
$$


(Although it is not relevant to our considerations, it is interesting to note that this is actually convergence with respect to a standard topology, the Vietoris finite topology, on $2^{A}$.) A function with domain $2^{A}$ will be called sequentially continuous if it preserves this type of sequential convergence.

Recall that the cardinal of $A$ is measurable if there exists a nonzero (completely additive) measure $\mu: 2^{A} \rightarrow 2$ (as usual 2 denotes the set $\{0,1\}$ with the discrete topology) which maps finite sets to zero. Temporarily, call a cardinal strongly sequential if there exists a nonzero sequentially continuous function $\sigma: 2^{A} \rightarrow 2$ mapping finite sets to zero. Although they are not given a name, strongly sequential cardinals are considered briefly in $[10$, p. 270] and our next result answers one of the questions posed there.

3.1 TheOREM. A cardinal is strongly sequential if and only if it is measurable.

Proof. Since each measure is sequentially continuous, each measurable cardinal is strongly sequential. Suppose $A$ has strongly sequential cardinality and let $\sigma: 2^{A} \rightarrow 2$ be as above. We first note that there exists a subset $A_{0}$ of $A$ such that $\sigma\left(A_{0}\right)=1$ and whenever $A_{0}=B \cup C$ with $B \cap C=\varnothing, \sigma(B)$ or $\sigma(C)$ is zero. (If not, we can construct disjoint sets $\left\{A_{n}\right\}$ with $A=\bigcup_{n} A_{n}$ and $\sigma\left(A_{n}\right)=1$ for each $n$. But then $\bigcup_{n} \bigcap_{m} A_{n+m}=\varnothing=\bigcap_{n} \bigcup_{m} A_{n+m}$ so $\sigma(\varnothing)=\lim _{n} \sigma\left(A_{n}\right)=1$ contrary to the assumption that $\sigma$ maps finite sets to zero.) Now define $\mu: 2^{A_{0}} \rightarrow 2$ by the rule: $\mu(B)=\max \left\{\sigma\left(B^{\prime}\right): B^{\prime} \subseteq B\right\}$; a straightforward computation shows that $\mu$ is a measure, so $A_{0}$ and hence $A$ has measurable cardinal.

Since the term is available, we call a cardinal squential if there exists a nonzero real valued sequentially continuous function $\sigma: 2^{A} \rightarrow R$ which maps finite sets to zero. It is shown in [12] that each cardinal less than the first weakly inaccessible cardinal is not sequential. Let II denote the space $\{0,1\}$ with topology $\{\varnothing,\{0,1\},\{1\}\}$, and let $R$ denote the real line.

3.2 TheORem. Let $X=\prod_{\alpha \in A} X_{\alpha}$ where each $X_{\alpha}$ is not indiscrete and, for a space $Z$, let $P(Z)$ denote the assertion: Each sequentially continuous function $f: X \rightarrow Z$ is 2-continuous. Then:

(i) $P(I I)$ if and only if $A$ is countable;

(ii) $P(R)$ if and only if card $A$ is not sequential;

(iii) $P(2)$ if and only if card $A$ is not measurable.

Proof. (i) If $A$ is uncountable, choose proper closed $Y_{\alpha} \subseteq X_{\alpha}$ and define $f(x)=0$ iff $\left\{\alpha: x_{\alpha} \notin Y_{\alpha}\right\}$ is countable. Then $f^{-1}(0)$ is proper, dense, and sequentially closed, so $f$ is sequentially continuous but not continuous. The converse is trivial.

(ii) Suppose card $A$ is sequential and suppose $\sigma: 2^{A} \rightarrow R$ is nontrivial and sequentially continuous. Define $f: \prod_{\alpha} X_{\alpha} \rightarrow R$ by the rule $f(x)=\sigma\left(\left\{\alpha: x_{\alpha} \notin Y_{\alpha}\right\}\right)$ where $Y_{\alpha}$ are as in (i). It is easily verified that $f$ is sequentially continuous but not continuous.

Now suppose card $A$ is not sequential, let $Y_{\alpha} \subseteq X_{\alpha}$ with $1 \leqq \operatorname{card}\left(Y_{\alpha}\right) \leqq 2$ for 
each $\alpha$, choose $x \in \prod_{\alpha} Y_{\alpha}$ and let $f: \prod_{\alpha} Y_{\alpha} \rightarrow R$ be sequentially continuous. By Theorem 2.1, the restriction of $f$ to the $\Sigma$-subspace $Y$ containing $x$ is continuous, so by for instance [5, Theorem 1] $\left.f\right|_{Y}$ extends to a continuous function $f^{*}: \prod_{\alpha} Y_{\alpha} \rightarrow R$. Choose any point $y$ in $\prod_{\alpha \in A} Y_{\alpha}$ and define $\sigma: 2^{A} \rightarrow R$ by the rule $\sigma(B)=f(x B y)$ $-f^{*}(x B y)$ where $x B y$ is the point with coordinates $y_{\alpha}$ for $\alpha$ in $B$ and $x_{\alpha}$ otherwise. Since $f$ and $f^{*}$ coincide on $Y, \sigma$ maps finite (indeed countable) sets to zero, and $\sigma$ is easily seen to be sequentially continuous. Since card $A$ is not sequential, it follows that $\sigma$ is identically zero, hence that $0=\sigma(A)=f(x A y)-f^{*}(x A y)=f(y)-f^{*}(y)$. Since $y$ was arbitrary, $f=f^{*}$ and is therefore continuous.

It should be mentioned that the proofs of this section are based on refinements of techniques introduced by Mazur in [12] and Varopoulos in [19]. Also, the statement in (ii) holds with $R$ replaced by any regular Hausdorff space $Z$ for which either each point of $Z$, or the diagonal of $Z \times Z$, is a sequential $G_{\delta}$, by essentially the same proof. This yields a corresponding improvement of Theorems 5.1 and 5.2. For results concerning ranges which are not regular, see [12] and [5].

4. Products of $k$-compact spaces. Where $Y$ is any space, let $k Y$ (resp. $k_{R} Y$ ) denote the set underlying $Y$ endowed with the smallest topology making each $k$-continuous (resp., real valued $k$-continuous) function on $Y$ continuous. Define $s Y$ and $s_{R} Y$ analogously, using sequentially continuous functions instead of $k$ continuous functions. Note that $Y$ is a $k$-space (resp. sequential space) if and only if $Y=k Y$ (resp. $Y=s Y$ ); we call a completely regular space $Y$ a $k_{R}$-space (resp. $s_{R}$-space) if $k_{R} Y=Y$ (resp. $s_{R} Y=Y$ ).

In contrast to $\$ \S 2$ and 3 , whose results will yield applications of Theorem 1.1, the results of this section will yield applications of Theorem 1.2. What we will need are conditions under which $k(X)$ or $s(X)$ will be countably compact, and conditions under which $k_{R}(X)$ and $s_{R}(X)$ will be pseudocompact, where $X$ is a product space $\prod_{\alpha \in A} X_{\alpha}$. We begin with a result for more general spaces. Call a subset $U$ of $X$ $k_{R}$-open, $s_{R}$-open, etc., if it is open in $k_{R} X, s_{R} X$, etc.

4.1 TheOREM. Let $X$ be any $T_{1}$ space.

(i) $k X$ is countably compact if and only if whenever $S \subseteq X$ is infinite, there exists a compact subset $K$ of $X$ such that $K \cap S$ is infinite.

(ii) $s X$ is countably compact if and only if $X$ is sequentially compact.

(iii) If $X$ is completely regular, $k_{R} X$ is pseudocompact if and only if for each infinite family of nonempty disjoint $k_{R^{-}}$open sets $\left\{U^{n}\right\}$, there exists a compact set $K \subseteq X$ such that $K \cap U^{n} \neq \varnothing$ for infinitely many $n$.

(iv) If $X$ is completely regular, $s_{R} X$ is pseudocompact if and only if for each infinite family of nonempty disjoint $s_{R}$-open sets $\left\{U^{n}\right\}$, there exists a convergent sequence $S \subseteq X$ such that $S \cap U^{n} \neq \varnothing$ for infinitely many $n$.

Proof. For (i) and (ii) recall that a $T_{1}$ space is countably compact if and only if each closed discrete subset is finite. A subset of $k X$ which meets each compact subset in a finite set, or a subset of $s X$ which meets each convergent sequence in a 
finite set, is closed and discrete. For (ii) and (iii), use complete regularity to construct a function $f: X \rightarrow R$ such that $f\left(X \backslash \bigcup_{n} U^{n}\right)=\{0\}$, while $f^{-1}(\{n\}) \subseteq U^{n}$ and $\left.f\right|_{U^{n}}$ is continuous, $n=1,2, \ldots$. If each compact set (resp. each convergent sequence) meets only finitely many of the $U^{n}, f$ is $k$-continuous (resp. squentially continuous). The converse direction is obvious.

We might mention that (i) and (iii) were proved (and used) in [15]. Suppose now that $X=\prod_{\alpha \in A} X_{\alpha}$. As examples in [8] show, the assumption that $k\left(X_{\alpha}\right)$ is countably compact for each $\alpha$ does not insure that $X$, much less $k X$, will be countably compact. However, if each $X_{\alpha}$ is strongly countably compact (each countable subset has compact closure) then $X$ is strongly countably compact, and therefore $k X$ is (strongly) countably compact. Regarding (ii), recall that $X=\prod_{\alpha \in A} X_{\alpha}$ is sequentially compact if and only if $A$ is countable and each factor is sequentially compact.

A space $Y$ such that $k_{R} Y$ is pseudocompact is said to be $k$-compact. This term was introduced in [4] where it is shown that $k$-compact spaces can, in the context of function spaces, serve as a satisfactory substitute for compact spaces. Nevertheless, the following analogue of Tychonoff's Theorem is surprising.

\subsection{THEOREM. Each product of completely regular $k$-compact spaces is $k$-compact.}

Proof. Let $X=\prod_{\alpha \in A} X_{\alpha}$ with each $X_{\alpha} k$-compact and suppose $X$ is not $k$ compact. Then there exists a countably infinite family, $\left\{U^{n}\right\}$, of nonempty disjoint $k_{R}$-open subsets of $X$ which has no cluster point. Choose $x^{n} \in U^{n}$ and $k_{R}$-open $V^{n}$ with $x^{n} \in V^{n}$ such that the $k_{R}$-closure of $V^{n}$ is contained in $U^{n}$. Then by Corollary 2.2 there exist finite $F_{n} \subseteq A$ such that $\Sigma_{F_{n}}\left(x^{n}\right) \subseteq V^{n}$ where $\Sigma_{B}(x)$ denotes the set of points $y$ in the $\Sigma$-subspace containing $x$ such that $y_{\alpha}=x_{\alpha}$ for each $\alpha$ in $B$. Thus the $k_{R}$-closure of each $\Sigma_{F_{n}}\left(x^{n}\right)$ is contained in $U^{n}$ so (as in the proof of Theorem 1.1) $\prod_{\alpha \in F_{n}}\left\{x_{\alpha}^{n}\right\} \times \prod_{\alpha \in A \mid F_{n}} X_{\alpha}$ is contained in $U^{n}$. Choose $x^{\prime} \in \prod_{\alpha \in A \mid C} X_{\alpha}$ where $C=\bigcup_{n} F_{n}$ and set $X^{\prime}=\prod_{\alpha \in C} X_{\alpha} \times\left\{x^{\prime}\right\}$. Then each $U^{n}$ meets $X^{\prime}$, so $X^{\prime}$ is not $k$-compact. Thus we may, without loss of generality, suppose that $A$ is countable. say $X=\prod_{i \in N} X_{i}$.

For each $n$, choose $k_{R}$-open sets $V_{i}^{n} \subseteq X_{i}, 1 \leqq i \leqq n$, such that

$$
\prod_{i=1}^{n} V_{i}^{n} \times \prod_{i>n}\left\{x_{i}^{n}\right\} \subseteq U^{n}
$$

This is possible since $k_{R}\left(\prod_{i=1}^{n} X_{i}\right)=\prod_{i=1}^{n} k_{R} X$ by [18, Theorem 4]. Now by Theorem 4.1 , if $U$ is any infinite family of nonempty disjoint $k_{R}$-open subsets of $X_{i}$, then there exists a compact subset $S$ of $X_{i}$ and an infinite subfamily $U^{\prime}$ of $U$ such that each member of $U^{\prime}$ meets $S$. Thus, inductively, we may choose infinite sets $N_{i} \subseteq$ $\{n: n \geqq i\}$ and compact subsets $K_{i}^{\prime}$ of $X_{i}$ such that $N_{i} \subseteq N_{i-1}$ and, for each $n \in N_{i}$, $K_{i}^{\prime} \cap V_{i}^{n} \neq \varnothing$. Choose $n_{i} \in N_{i}$, note that $\left\{n_{i}\right\}$ is infinite, and set

$$
K_{i}=K_{i}^{\prime} \cup\left\{\left(x^{n}\right)_{i}: j<i\right\} .
$$


Then each $K_{i}$ is compact, so $K=\prod_{i} K_{i}$ is a compact subset of $X$. To complete the proof (that is, to contradict the assumption that $\left\{U^{n}\right\}$ has no cluster point) it suffices to show that $K \cap U^{n_{j}} \neq \varnothing$ for each $j$. For a fixed index $j$, define $y^{j}$ as follows: For $i>j,\left(y^{j}\right)_{i}=\left(x^{n}\right)_{i}$; for $i \leqq j\left(y^{j}\right)_{i} \in K_{i} \cap V_{i}^{n_{3}}$ (this intersection is not empty since $\left.n_{j} \in N_{j} \subseteq N_{i}\right)$. Now $y^{j} \in K$ since, by the construction of $K_{i}$, $\left(y^{j}\right)_{i}$ $=\left(x^{n_{j}}\right)_{i} \in K_{i}$ for $j<i$. Finally, $y^{j} \in U^{n_{j}}$, in fact, $y^{j} \in \prod_{i=1}^{n_{j}} V_{i}^{n_{j}} \times \prod_{i>n_{j}}\left\{\left(x^{n_{j}}\right)_{i}\right\}$, since $j \subseteq n_{j}$ (because $n_{j} \in N_{j} \subseteq\{n: n \geqq j\}$ ).

We naturally call a space $Y s$-compact if $s_{R} Y$ is pseudo-compact.

4.3 THEOREM. Let $X=\prod_{\alpha \in A} X_{\alpha}$ where each $X_{\alpha}$ is completely regular and scompact. If card $(A)$ is not sequential, $X$ is s-compact.

Proof. Since card $(A)$ is not sequential, the reduction to the case with $A$ countable goes through as in the proof of Theorem 4.2. Paralleling that proof, one constructs points $x^{n}$ in $U^{n} \cap K$ where $K$ is a product of countably many convergent sequences. Since $K$ is compact and first countable, it is sequentially compact so $\left\{x^{n}\right\}$ have a convergent subsequence, as desired.

For our applications we need results slightly stronger than 4.2 and 4.3. The proofs of these two theorems easily generalize to yield:

4.4 TheOREM. Let $X=\prod_{\alpha} X_{\alpha}$ where each $X_{\alpha}$ is completely regular, let $Y_{\alpha} \subseteq X_{\alpha}$ and let $Y=\prod_{\alpha} Y_{\alpha}$.

(i) If each $Y_{\alpha}$ is pseudocompact as a subspace of $k_{R}\left(X_{\alpha}\right)$, then $Y$ is pseudocompact as a subspace of $k_{R}(X)$.

(ii) If card $A$ is not sequential and each $Y_{\alpha}$ is pseudocompact as a subspace of $s_{R}\left(X_{\alpha}\right)$, then $Y$ is pseudocompact as a subspace of $s_{R}(X)$.

5. Sequentially continuous and $k$-continuous functions on products. The problem of determining when finite products of $k$-spaces, $k_{R}$-spaces, sequential spaces or $s_{R}$-spaces will retain these properties has been studied in [14], [18], [20], and the papers referenced therein. In particular, the results of [18] show that for $X_{0}$ as in the discussion following 2.2 and, for $n \geqq 1, X_{n}$ a copy of the integers, $\prod_{n=0}^{\infty} X_{n}$ is not a $k_{R}$-space even though each finite subproduct is sequential (and $\prod_{n=1}^{\infty} X_{n}$ is first countable).

Turning to infinite products, we begin with some additional terminology. Call a space $Y$ a $k_{3}$-space if each $k$-continuous function on $Y$ with regular (or equivalently $T_{3}$ ) range is continuous, and an $s_{N}$-space if each integer valued sequentially continuous function on $Y$ is continuous.

5.1 Theorem. Let $X=\prod_{\alpha \in A} X_{\alpha}$ where each $X_{\alpha}$ is not indiscrete.

(i) If $X$ is sequential, then card $(A)$ is countable.

(ii) If $X$ is an $\dot{s}_{R}$-space, then card $(A)$ is not sequential.

(iii) If $X$ is an $s_{N}$-space, then card $(A)$ is not measurable.

Furthermore, if each $X_{\alpha}$ is of type $\mathscr{C}^{*}$, the converse of each of these statements is true. 
Proof. Theorems 1.1, 2.3, 2.4 and 3.2.

5.2 TheOREM. Let $X=\prod_{\alpha \in A} X_{\alpha}$ where each $X_{\alpha}$ is a completely regular $s_{R}$-space. If all but one of the $X_{\alpha}$ are pseudocompact, then $X$ is an $s_{R}$-space if and only if card $(A)$ is not sequential.

Proof. Suppose that for $\alpha \neq \alpha_{0}, X_{\alpha}$ is pseudocompact. Let $f: X \rightarrow R$ be sequentially continuous, and let $F \subseteq A$ be finite. We will be applying Theorem 1.2, and an examination of the proof of that theorem shows that we may suppose that $\alpha_{0} \in F$. Now for $X^{\prime}=s_{R}\left(\prod_{\alpha \in A \mid F} X_{\alpha}\right)$ and $X^{\prime \prime}=\prod_{\alpha \in F} X_{\alpha}, X^{\prime \prime}$ is a sequential space by [18, Theorem 4] and $X^{\prime}$ is pseudocompact by Theorem 4.3. Hence by [17, Corollary 1] $\pi_{F}: X^{\prime} \times X^{\prime \prime} \rightarrow X^{\prime \prime}$ maps zero sets to zero sets. Now by [18, Theorem 4], $X^{\prime} \times X^{\prime \prime}$ is itself an $s_{R}$-space, so $f: X^{\prime} \times X^{\prime \prime} \rightarrow R$ is continuous. It follows that $\pi_{F} \circ f^{-1}$ is closed. Hence by Theorem $1.2, X$ is an $s_{R}$-space.

Theorem 5.2 is best in the sense that if infinitely many of the $X_{\alpha}$ are not pseudocompact, then there exists an $s_{R}$-space $X_{\alpha_{0}}$ such that $\prod_{\alpha \in A \cup\left\{\alpha_{0}\right\}} X_{\alpha}$ is not an $s_{R}$-space. (This follows by [18, Theorem 4].) However, if we restrict every factor, a somewhat sharper result is possible.

5.3 THEOREM. Let $X=\prod_{\alpha \in A} X_{\alpha}$ where each $X_{\alpha}$ is a completely regular $s_{R}$-space. If each $X_{\alpha}$ is locally pseudocompact, then $X$ is an $s_{R}$-space if and only if card $(A)$ is not sequential.

Proof. Let $f: X \rightarrow R$ be sequentially continuous, let $U \subseteq R$ be open, let $x \in f^{-1}(U)$ and let $V$ be a neighborhood of $f(x)$ such that cl $V \subseteq U$. For each $\alpha$ let $S_{\alpha}$ be a closed pseudocompact neighborhood of $x_{\alpha}$. As in the proof of Theorem 5.2 (the necessary result on projections is given in [17, Theorem 2]) the restriction of $f$ to $S=\prod_{\alpha \in A} S_{\alpha}$ is continuous, so we may suppose that $S$ is contained in $f^{-1}(V)$. For $F \subseteq A$ let $\Sigma_{F}(S)=\left\{x \in X\right.$ : for each $\alpha$ in $F, x_{\alpha} \in S_{\alpha}$, and $x_{\alpha} \notin S_{\alpha}$ for only finitely many $\alpha$ in $A\}$. It suffices to show that $\Sigma_{F}(S)$ is contained in $f^{-1}(\mathrm{cl} V)$ for some finite $F$ (since then $\operatorname{cl} \Sigma_{F}(S) \subseteq f^{-1}(U)$ is a neighborhood of $X$ ). Suppose not; then we can construct a sequence $\left\{V^{n}\right\}$ of $k_{R}$-open sets such that

$$
V^{n} \subseteq \prod_{\alpha \in A^{\prime}} S_{\alpha} \times \prod_{\alpha \in F_{n}}\left(X_{\alpha} \mid S_{\alpha}\right) \times \prod_{\alpha \in A^{\prime \prime}} X_{\alpha}
$$

where $A^{\prime}=\bigcup_{i=1}^{n-1} F_{i}, A^{\prime \prime}=A \mid\left(A^{\prime} \cup F_{n}\right)$ and $F_{n}$ is finite and disjoint from $A^{\prime}$, such that $V^{n} \cap f^{-1}(V)=\varnothing$ for each $n$. Choose $x^{n} \in V^{n}$ and set $S_{\alpha}^{\prime}=S_{\alpha} \cup\left\{x_{\alpha}^{n}\right\}$. Each $S_{\alpha}^{\prime}$ is pseudocompact (since $S_{\alpha}^{\prime} \mid S_{\alpha}$ contains at most one point) so $\prod_{\alpha} S_{\alpha}^{\prime}=S^{\prime}$ is pseudocompact when topologized as a subspace of $s_{R}(X)$ (by Theorem 4.4). Hence $\left\{V^{n} \cap S^{\prime}\right\}$ must have a $k_{R}$-cluster point, and clearly that cluster point is in $S$. This contradicts the fact that $f^{-1}(U)$ is $k_{R}$-open and completes the proof.

Recall that each $T_{0}$ topological group is completely regular Hausdorff. Call a group $G$ an $s$-group (resp. $k$-group) if each sequentially continuous (resp. $k$ continuous) homomorphism from $G$ to a $T_{0}$ group is continuous. By Theorems 1.3, 2.2, and 3.2 we have: 
5.4 TheOREM. Let $G=\prod_{\alpha \in A} G_{\alpha}$, let $G_{0}=\{x \in G: \operatorname{card}(\delta(x, e))$ is not sequential $\}$ (where $e$ is the identity of $G$ ) and let $H$ be any subgroup of $G_{0}$ which is invariant under projections. If each $G_{\alpha}$ is an s-group, then $H$ is an s-group.

Note that (also by Theorems 1.3 and 3.2 ) the property "each sequentially continuous homomorphism whose range has a base at the identity consisting of open subgroups is continuous" is preserved under products with nonmeasurably many factors. The corresponding result for homomorphisms whose range contains no small subgroups (for instance, any Lie group) also holds, by an easy adaptation of the proof of $\left[19\right.$, Lemma $\left.\pi_{2}\right]$. For some much more interesting results concerning sequentially continuous homomorphisms, see [19].

Turning now to $k$-continuous functions, note that by [7, Theorem 4.3] and Theorem 4.1, countably compact $k$-spaces are preserved under countable products, and by Proposition 2.3 spaces of type $\mathscr{C}$ are preserved under countable products.

5.5 Proposition. Suppose each $X_{\alpha}$ is a $T_{1} k$-space. If $\prod_{\alpha \in A} X_{\alpha}$ is a k-space, then all but countably many of the $X_{\alpha}$ are countably compact, and the product of the countably compact factors is pseudocompact.

Proof. A straightforward adaptation of the proof outlined in [11, p. 240] shows that a product of uncountably many infinite discrete spaces is not a $k$-space. Since a $T_{1}$-space is countably compact if and only if it contains no infinite closed discrete subspace, and since each closed subspace of a $k$-space is a $k$-space, it follows that all but countably many of the factors are countably compact. Now a product space is pseudocompact if each of its countable subproducts is pseudocompact, so the product of the countably compact factors is pseudocompact.

5.6 THEOREM. Let $X=\prod_{\alpha \in A} X_{\alpha}$.

(i) If each $X_{\alpha}$ is of type $\mathscr{C}$, then $X$ is a $k_{3}$-space.

(ii) If each $X_{\alpha}$ is a completely regular locally pseudocompact $k_{R^{-}}$space, then $X$ is a $k_{R}$-space.

Proof. (i) Follows by Theorems 1.1 and 2.4, and (ii) follows by the obvious adaptation of the proof of 5.3.

5.7 THEOREM. If $H$ is a subgroup of a product of $k$-groups and $H$ is invariant under projections, then $H$ is a $k$-group.

Proof. Theorems 1.3 and 2.2 .

\section{REFERENCES}

1. A. V. Arhangel'skiĭ, Bicompact sets and the topology of spaces, Trudy Moskov. Mat. Obšč. 13 (1965), 3-55 = Trans. Moscow Math. Soc. 1965, 1-62. MR 33 \#3251.

2. W. W. Comfort, On the Hewitt realcompactification of a product space, Trans. Amer. Math. Soc. 131 (1968), 107-118. MR 36 \#5896.

3. H. H. Corson, Normality in subsets of product spaces, Amer. J. Math. 81 (1959), 785-796. MR 21 \#5947.

4. — Compact subsets and the C. O. Topology (to appear). 
5. R. Engelking, On functions defined on Cartesian products, Fund. Math. 59 (1966), 221-231. MR 34 \#3546.

6. I. Fleischer and S. P. Franklin, "On compactness and projections" in Contributions to extension theory of topological structures, Academic Press, New York, 1969, 77-79.

7. Z. Frolik, The topological product of two pseudo-compact spaces, Czechoslovak Math. J. 10 (85) (1960), 339-349. MR 22 \#7099.

8. - The topological product of countably compact spaces, Czechoslovak Math. J. 10 (85) (1960), 329-338. MR 22 \#480.

9. A. Hager, Projections of zero sets (and the fine uniformity on a product), Trans. Amer. Math. Soc. 140 (1969), 87-94.

10. H. J. Keisler and A. Tarski, From accessible to inaccessible cardinals. Results holding for all accessible cardinal numbers and the problem of their extension to inaccessible ones, Fund. Math. 53 (1963/64), 225-308. MR 29 \#3385.

11. J. L. Kelley, General topology, Van Nostrand, Princeton, N. J., 1955. MR 16, 1136.

12. S. Mazur, On continuous mappings on Cartesian products, Fund. Math. 39 (1952), 229-338. MR 14, 1107.

13. E. A. Michael, $\boldsymbol{\aleph}_{0}$-spaces, J. Math. Mech. 15 (1966), 983-1002. MR 34 \#6723.

14. - Local compactness of Cartesian products of quotient maps and $k$-spaces, Ann. Inst. Fourier Grenoble 53 (1969), 281-286.

15. N. Noble, Countably compact and pseudocompact products, Czechoslovak Math. J. 19 (94) (1969), 390-397.

16. - Products with closed projections, Trans. Amer. Math. Soc. 140 (1969), 381-391.

17. - A note on z-closed projections, Proc. Amer. Math. Soc. 23 (1969), 73-76.

18. - Products of quotient maps and spaces with weak topologies, (to appear).

19. N. Varopoulos, A theorem on the continuity of homomorphisms of locally compact groups, Proc. Cambridge Philos. Soc. 60 (1964), 449-463. MR 29 \#184.

20. D. Weddington, $k$-spaces, Doctoral Dissertation, University of Miami, Coral Gables, Fla., 1968.

Clark University,

WORCESTER, MASSACHUSETTS 01610 